\title{
Robust Interference Exploitation for Multi-Cell Transmission
}

\author{
Zhongxiang Wei, Christos Masouros, Tongyang Xu, and Kai-Kit Wong \\ Department of Electronic and Electrical Engineering \\ University College London, London, UK \\ \{zhongxiang.wei, c.masouros, tongyang.xu.11, kai-kit.wong\}@ucl.ac.uk
}

\begin{abstract}
In this paper, we investigate power-efficient constructive interference (CI) exploitation in multi-cell coordination systems. By only sharing channel state information (CSI) among the coordinated base stations (BS)s, we propose a CI-based coordinated beamforming (CBF) scheme to judiciously exploit multiuser interference as a beneficial element rather than strictly mitigating it, while simultaneously suppressing inter-cell interference as a destructive element. Then taking imperfect channel state information (CSI) into consideration, we minimize the total transmission power consumption with multiple users' probabilistic signal-to-interference-and-noise ratio (SINR) requirements, where the users' SINR requirements are guaranteed in a statistical manner. Finally, under the presence of CSI error, simulation results demonstrate that the proposed CI-based CBF scheme consumes much lower transmission power compared to the classical CBF benchmarks, where both intra-cell multi-user and inter-cell interference need to be strictly cancelled as destructive elements. Last but not least, the incurred overhead and computational complexity of the proposed scheme are analytically analyzed, confirming its practicality as a new dimension on multi-cell coordination.

Index Terms-Constructive Interference Exploitation, Probabilistic Robust Optimizations, Multi-Cell Coordination.
\end{abstract}

\section{INTRODUCTION}

Inter-cell and intra-cell multi-cell interference have long been considered as the most harmful elements impairing the system performance [1]. With the upcoming ultra-dense or heterogeneous networks, the management of the ubiquitous interference has presented great challenges for researchers. In the past decades, coordinated multi-point (CoMP) systems have attracted much attention in academia [2]. By sharing the channel state information (CSI) and the data to be transmitted, the coordinated base station (BS)s are transformed into a distributed multiple-input multiple out-put (MIMO) system, where inter-cell channels are enabled to carry useful information. To this end, optimal channel and power allocation schemes were investigated for CoMP systems in [3]. In [4], cooperative beamforming among the coordinated BSs was designed for CoMP systems at downlink, considering the effect of imperfect CSI. In [5], energy efficiency maximization in CoMP systems was investigated, where cooperative beamforming was designed with the consideration of low power consumption. From the perspective of information theory, CoMP can be considered as a virtual MIMO system, where BSs exactly act as distributed antennas for joint transmission. Nevertheless, CoMP incurs tremendous overhead for multi-cell coordination, and all the coordinated BSs need to be connected with high-speed and delay-free back-hauls for global CSI and data sharing, which may not be always available in practice.

In this spirit, a low overhead multi-cell coordination scheme, namely coordinated-beamforming $(\mathrm{CBF})$, has been extensively researched [6]. By $\mathrm{CBF}$ scheme, only CSI is required to be exchanged among the coordinated BSs. Since each BS is unaware of the transmitted data of adjacent cells, intercell gain in this scenario needs to be strictly mitigated by the joint beamforming design. Based on the design principle, [7] and [8] investigated power minimization problems under users' SINR requirements. Differently, the authors in [9] and [10] maximized the overall downlink and uplink throughput performance, where BS only transmits or decodes the signal from the users within its cell while treating the inter-cell interference as harmful noise. In summary, CBF only needs to share CSI among the coordinated BSs, and hence effectively reduces the incurred overhead compared to CoMP systems. By cooperative beamforming design, it strikes a good tradeoff between optimizing the SINR to/from the user within the cell of interest and suppressing inter-cell interference.

Note that, regardless of CoMP or CBF, multi-user interference should be always strictly suppressed. However, based on the recently introduced constructive interference (CI) exploitation, there is a possibility to utilize multi-user interference as a source of useful signal. By doing so, less transmission power is consumed to achieve a target SINR, as multi-user interference is effectively utilized rather being mitigated. CI was initially presented in code division multiple access systems [11]. Later on, CI was investigated in precoding design of multiuser MIMO systems [12], physical layer security systems [13], cognitive radio [14], and Internet-of-Things [15]. However, it is aware that the existing researches on CI only focus on single-cell design, which may not be trivially extended to $\mathrm{CBF}$ systems. It is because based on the principle of CI, there is scope to exploit intra-cell multiuser interference as constructive elements. Nevertheless by CBF, each BS is unaware of the data transmitted by other adjacent BSs, which makes exploiting inter-cell interference as constructive elements impossible. Hence, how to judiciously design CI precoding to utilize intracell multiuser interference while simultaneously suppressing 
inter-cell interference is still unknown. In addition, the globally shared CSI may be imperfect or out-of-dated, and thus the presence of CSI uncertainty makes CI design in CBF more challenging.

Motivated by the open challenges, we aim to research robust CI precoding design under imperfect CSI acquisitions, where intra-cell (multi-user) interference is effectively utilized as a green source of signal while inter-cell interference is strictly suppressed. Our contributions are summarized as follows:

1) We first propose a novel CI design for the CBF multi-cell coordination scenario, which is fundamentally different from the existing single-cell $\mathrm{CI}$ exploitation and the classical interference avoidance based CBF multi-cell coordination. Explicitly, based on the low overhead of only sharing CSI, we investigate the geometrical interpretation of the CI design to fully utilize multi-user interference as a constructive element while simultaneously suppressing inter-cell interference.

2) Then, a robust power-efficient CI precoding design is proposed to minimize total transmit power subject to users' probabilistic SINR constraints, under the presence of CSI uncertainties. Explicitly, multiple users' SINR requirements are statistically guaranteed and the probability of satisfying SINR requirements is maintained to be higher than a pre-set threshold.

3) Finally, the incurred overhead of the proposed CI-based coordination scheme is analyzed. It is confirmed that the proposed design requires the same level of overhead with conventional CBF schemes, while maintaining the advantage of utilizing multi-user interference. Also, the complexity is analytically calculated, demonstrating a good trade-off between power efficiency performance and practicality.

Notations: $\|\cdot\|_{p}$ denotes the p-norm of a vector. $\boldsymbol{A}^{H}, \operatorname{Tr}(\boldsymbol{A})$ and $\operatorname{Rank}(\boldsymbol{A})$ represent the Hermitian transpose, trace, and rank of a matrix $\boldsymbol{A} . \boldsymbol{A} \succeq 0$ means $\boldsymbol{A}$ is a positive semi-definite matrix. Superscript $\Re$ and $\Im$ denote the real and imaginary parts of a complex variable, respectively. $\boldsymbol{I}_{n}$ denotes an $n$-by$n$ identity matrix.

\section{System Model And Constructive InTERference}

In this section, the system model is first introduced, and then the fundamentals of CI design are presented.

\section{A. System Model}

A CBF system at downlink is considered, where $N_{B S}$ BSs share CSI for multi-cell coordination and there are $K$ users in each cell. Each BS is equipped with $M$ antennas, while each user is equipped with single antenna for simplicity. Define $\mathrm{U}_{i k}$ as the $k$-th user located within the $i$-th cell, and $\bar{\Gamma}_{i k}$ as the preset SINR requirement of the user $\mathrm{U}_{i k}$. The wireless channel ranging from the $i$-th BS to the user $\mathrm{U}_{i k}$ is given as $\boldsymbol{g}_{i i k}=$ $\hat{\boldsymbol{g}}_{i i k}+\boldsymbol{e}_{i k}$, where $\hat{\boldsymbol{g}}_{i i k} \in \mathbb{C}^{M \times 1}$ denotes the estimated CSI with uncertainties $\boldsymbol{e}_{i k} \in \mathbb{C}^{M \times 1}, \forall k \in K$, and $i \in N_{B S}$. In practice, CSI estimation and sharing of CBF systems can be performed in the training phase [7]. By classic unbiased estimators, such as minimum mean square error channel estimator, the element of CSI error vector can be modeled to follow Gaussian distribution as $\left[\boldsymbol{e}_{i k}\right]_{m} \sim \mathbb{C N}\left\{0, \sigma_{i k}^{2}\right\}, \forall m \in M$ [17]. Hence, the composite signal at the user $\mathrm{U}_{i k}$ is calculated as

$$
r_{i k}=\boldsymbol{g}_{i i k}^{T} \sum_{n=1}^{K} \boldsymbol{w}_{i n} x_{i n}+\sum_{j \neq i}^{N_{B S}} \sum_{m=1}^{K} \boldsymbol{g}_{j i k}^{T} \boldsymbol{w}_{j m} x_{j m}+n_{i k},
$$

where $\boldsymbol{w}_{i n} \in \mathbb{C}^{M \times 1}$ and $x_{i n}$ represent the precoding and the data to be transmitted at the $i$-th BS for $\mathrm{U}_{i n} . n_{i k} \in \mathbb{C}$ represents the additive white Gaussian noise (AWGN) following $n_{i k} \sim \mathbb{C N}\left(0, \sigma_{n}^{2}\right), \forall \mathrm{U}_{i k}$. By conventional CBF, the instantaneous SINR is given as

$$
\begin{aligned}
& \Gamma_{i k}= \\
& \frac{\left|\boldsymbol{h}_{i i k} \boldsymbol{w}_{i k}\right|^{2}}{\sum_{k^{\prime} \neq k, k^{\prime} \in i}\left|\boldsymbol{g}_{i i k} \boldsymbol{w}_{i k^{\prime}}\right|^{2}+\sum_{j \neq i}^{N_{B S}} \sum_{m=1}^{K}\left|\boldsymbol{g}_{j i k} \boldsymbol{w}_{j m}\right|^{2}+\sigma_{n}^{2}},
\end{aligned}
$$

which suggests the effect of multiuser and inter-cell interference (in the first and second terms of the denominator) should be strictly mitigated to improve the user's SINR.

\section{B. Constructive Interference}

By conventional CBF and CoMP schemes, the transmitted signal is considered as an infinite Gaussian signal. Hence, any interference adds perturbation to the signal and harms performance. Following this principle, multi-user interference should be always strictly cancelled, and one needs to contain the received symbols within a region around the nominal point in the signal constellation, as shown in Fig. 1(a). Nevertheless, since the transmitted symbols are available at the local BS, it is judicious to jointly utilize the spatial correlation among the channels and the symbols to be transmitted. That is, the interference can be exploited as a desired element to push the received signals away from the detection thresholds of the signal constellation. In this case, higher degrees-of-freedom (DoF) at transmitter side is obtained, and the increased distance to the detection threshold can benefit the receiver side performance.

Without loss of generality, assume that the intended symbol is $(1+i) / \sqrt{2}$ with QPSK modulation. By CI design shown in Fig. 1(b), the received signal $r_{i k}$ can be pushed into a constructive region (green area), rather than being strictly located in the proximity region around the constellation point. To guarantee the constructive effect of the interference, geometric interpretation can be exploited as shown in Fig. 1(c). Explicitly, we can rotate the received signal by an angle $\angle x_{i k}^{*}$, and the rotated signal (noise excluded) can be mapped onto real axis $\alpha_{R}=\Re\left\{r_{i k} x_{i k}^{*}\right\}$ and imaginary axis $\alpha_{I}=\Im\left\{r_{i k} x_{i k}^{*}\right\}$, respectively. As can be seen, the received signal falls into a constructive region (in Fig. 1 (b)) if and only if the trigonometry $\left|\alpha_{I}\right| \leq\left(\alpha_{R}-\gamma\right) \tan \theta$ (in Fig. 1(c)) holds, where $\theta=\frac{\pi}{L}$ and $L$ represents constellation size. The above discussion can be extended into any order L-PSK and multi-level modulations. For brevity we refer the readers to [16] for details. 

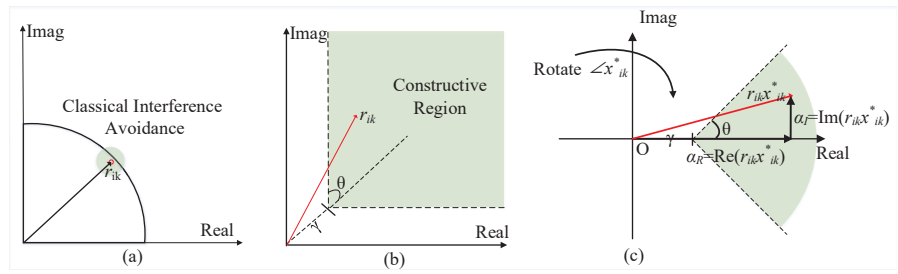

Fig. 1. The geometrical interpretation of CI design, where the intended symbol is $(1+i) / \sqrt{2}$ with QPSK modulation for example. (a) Conventional precoding result in a proximity region around the constellation point. (b) By CI design, the received signal $r_{i k}$ falls into a constructive region (green area), where $\gamma=\sigma_{n} \sqrt{\bar{\Gamma}_{i k}}$. (c) After being rotated by $\angle x_{i k}^{*}$, the received signal falls into constructive region as long as the inequality $\left|\alpha_{I}\right| \leq\left(\alpha_{R}-\gamma\right) \tan \theta$ holds, where the SINR requirement $\bar{\Gamma}_{i k}$ is also naturally guaranteed.

\section{Power-EFFicient Robust CI Design FOR CBF}

In this section, we investigate the power-efficient robust precoding optimization for CBF coordination, under imperfect CSI acquisitions.

\section{A. Constructive Multi-user Interference Exploitation in $C B F$}

Since the intended symbol can be written as $x_{i k}=$ $d_{i k} e^{j\left(\phi_{i k}\right)}$, we can express the symbol $x_{i k}$ by another symbol $x_{i n}$ as $x_{i n}=x_{i k} e^{j\left(\phi_{i n}-\phi_{i k}\right)}$. Note that the selection of the reference symbol $x_{i n}$ can be arbitrary. Hence, (1) is written as

$$
r_{i k}=\boldsymbol{g}_{i i k}^{T} \sum_{n=1}^{K} \boldsymbol{w}_{i n} e^{j\left(\phi_{i n}-\phi_{i k}\right)} x_{i k}+\sum_{j \neq i}^{N_{b s}} \sum_{m=1}^{K} \boldsymbol{g}_{j i k}^{T} \boldsymbol{w}_{j m} x_{j m}+n_{i k}
$$

Define $\tilde{\boldsymbol{g}}_{i i k}=\boldsymbol{g}_{i i k} e^{j\left(\phi_{i 1}-\phi_{i k}\right)}$ and $\tilde{\boldsymbol{g}}_{j i k}=\boldsymbol{g}_{j i k} e^{j\left(\phi_{j 1}-\phi_{j m}\right)}$ as the equivalent channels. Define $\boldsymbol{w}_{i}=\sum_{n=1}^{K} w_{i n} e^{j\left(\phi_{i n}-\phi_{i 1}\right)}$ and $\boldsymbol{w}_{j}=\sum_{m=1}^{K} w_{j n} e^{j\left(\phi_{j n}-\phi_{j 1}\right)}$ as the overall precoding vectors at the $i$-th and $j$-th $\mathrm{BSs}$, respectively. Hence, (3) can be simplified into

$$
r_{i k}=\tilde{\boldsymbol{g}}_{i i k}^{T} \boldsymbol{w}_{i} x_{i k}+\sum_{j \neq i}^{N_{B S}} \tilde{\boldsymbol{g}}_{j i k}^{T} \boldsymbol{w}_{j} x_{j m}+n_{i k},
$$

which suggests that the CI design transforms the broadcast channel in each cell into a virtual multicast channel with common messages to all local users. In other word, the intracell multi-user interference can be utilized as a useful signal to enhance the receiving performance. Accordingly, we have the following inequality to guarantee the constructive effect of multi-user interference.

$$
\left|\Im\left(\sum_{j=1}^{N_{B S}} \tilde{\boldsymbol{g}}_{i i k}^{T} \boldsymbol{w}_{i}\right)\right| \leq\left(\Re\left(\sum_{j=1}^{N_{B S}} \tilde{\boldsymbol{g}}_{i i k}^{T} \boldsymbol{w}_{i}\right)-\sqrt{\left(\sigma_{n}^{2}+\zeta_{i k}\right) \bar{\Gamma}_{i k}}\right) \tan \theta
$$

where $\zeta_{i k}=\sum_{j \neq i}^{N_{B S}}\left\|\tilde{\boldsymbol{g}}_{j i k}^{T} \boldsymbol{w}_{j}\right\|^{2}$ denotes the inter-cell interference. Under the geometrical provision of (5), generating constructive multi-user interference and satisfying the user's SINR requirement are simultaneously guaranteed. Nevertheless, note that inter-cell interference should be still suppressed as noise, which is fundamentally different from that in single-cell CI designs [12]- [16].

\section{B. Problem Formulation}

Under the aforementioned CI exploitation, we aim to minimize transmission power consumption, subject to multiple constraints. The problem is formulated as

$$
\begin{aligned}
& P 1(\mathrm{CI}-\mathrm{CBF}-\text { Robust }): \underset{\boldsymbol{w}_{i}, \forall i \in \mathbb{N}_{B S}}{\operatorname{argmin}} \sum_{j=1}^{N_{B S}}\left\|\boldsymbol{w}_{i}\right\|_{2}^{2}, \\
& \text { s.t }(C 1):\left\|\boldsymbol{w}_{i}\right\|^{2} \leq P_{\max }, \forall j \in \mathbb{N}_{B S}, \\
& \quad(C 2): \operatorname{Pr}\left\{\Gamma_{i k} \geq \bar{\Gamma}_{i k} \mid \boldsymbol{e}_{i k}\right\} \geq \eta_{i k}, \forall \mathrm{U}_{i k} .
\end{aligned}
$$

where $(\mathrm{C} 1)$ constrains the dissipated transmission power at each BS to be lower than its budget $P_{\max }$. (C2) means that the probability of satisfying the user's SINR requirement should be higher than a preset threshold $\eta_{i k}, \forall \mathrm{U}_{i k}$.

\section{Optimization Solution}

The difficulty of solving P1 lies in its probabilistic constraint (C2), under infinite possibilities of CSI uncertainties. Based on the geometrical interpretation in (5), the probabilistic constraint (C2) can be expanded into the two inequalities in (7). To handle the inequalities, we first introduce two auxiliary variables $\varphi_{i k}$ and $u_{i k}$ to decouple the inter-cell interference at the right hand of (7). Now, (7) is equivalent to the four inequalities in (8), which are handled one by one.

For constraint (C2a), we first collect the CSI related elements into another long vector such that $\boldsymbol{f}_{j i k}=\left[\hat{\boldsymbol{g}}_{i i k}^{\Im}+\boldsymbol{e}_{i k}^{\Im}-\right.$ $\left.\hat{\boldsymbol{g}}_{i i k}^{\Re} \tan \theta-\boldsymbol{e}_{i k}^{\Re} \tan \theta ; \hat{\boldsymbol{g}}_{i i k}^{\Re}+\boldsymbol{e}_{i k}^{\Re}+\hat{\boldsymbol{g}}_{i i k}^{\Im} \tan \theta+\boldsymbol{e}_{i k}^{\Im} \tan \theta\right]$. It is found that the vector $\boldsymbol{f}_{j i k}$ 's expection is $\overline{\boldsymbol{f}}_{i k}=\left[\hat{\boldsymbol{g}}_{i i k}^{\Im}-\right.$ $\left.\hat{\boldsymbol{g}}_{i i k}^{\Re} \tan \theta ; \hat{\boldsymbol{g}}_{i i k}^{\Re}+\hat{\boldsymbol{g}}_{i i k}^{\Im} \tan \theta\right]$ with covariance matrix $\boldsymbol{\Lambda}_{i k}=$ $\operatorname{diag}(\underbrace{\left(1+\tan ^{2} \theta\right) \sigma_{i k}^{2}, \ldots,(1+\tan \theta)^{2} \sigma_{i k}^{2}}_{2 M})$. Hence, we have

$$
\begin{aligned}
& \operatorname{Pr}\left\{\frac{\left(\boldsymbol{f}_{i k}^{T}-\overline{\boldsymbol{f}}_{i k}^{T}\right)\left[\boldsymbol{w}_{i}^{\Re} ; \boldsymbol{w}_{i}^{\Im}\right]}{\left\|\boldsymbol{\Lambda}_{i k}^{\frac{1}{2}}\left[\boldsymbol{w}_{i}^{\Re} ; \boldsymbol{w}_{i}^{\Im}\right]\right\|_{2}} \leq\right. \\
&\left.\frac{-\left(\sigma_{n}+\varphi_{i k}\right) \sqrt{\overline{\Gamma_{i k}}} \tan \theta-\overline{\boldsymbol{f}}_{i k}^{T}\left[\boldsymbol{w}_{i}^{\Re} ; \boldsymbol{w}_{i}^{\Im}\right]}{\left\|\boldsymbol{\Lambda}_{i k}^{\frac{1}{2}}\left[\boldsymbol{w}_{i}^{\Re} ; \boldsymbol{w}_{i}^{\Im}\right]\right\|_{2}}\right\} \geq \eta_{i k} .
\end{aligned}
$$

which acts as the cumulative probability function (cdf) of a standard Gaussian variable. Define $\Phi(x)$ as cdf of a standard Gaussian variable and $\Phi^{-1}(\cdot)$ as the its inverse function. (9) can be given as a quadratic inequality

$$
\begin{aligned}
(C 2 a): \overline{\boldsymbol{f}}_{i k}^{T}\left[\boldsymbol{w}_{i}^{\Re} ; \boldsymbol{w}_{i}^{\Im}\right]+\Phi^{-1}\left(\eta_{i k}\right) \| & \boldsymbol{\Lambda}_{1}^{\frac{1}{2}}\left[\boldsymbol{w}_{i}^{\Re} ; \boldsymbol{w}_{i}^{\Im}\right] \|_{2} \leq \\
& -\left(\varphi_{i k}+\sigma_{n}\right) \sqrt{\bar{\Gamma}_{i k}} \tan \theta .
\end{aligned}
$$

According to Schur complement, constraint (C2a) can be finally transformed into a linear matrix inequality (LMI) in (11). Similarly, constraint (C2b) can be given in (12), where $\overline{\boldsymbol{d}}_{i k}=$ $\left[-\hat{\boldsymbol{g}}_{i i k}^{\Im}-\hat{\boldsymbol{g}}_{i i k}^{\Re} \tan \theta ;-\hat{\boldsymbol{g}}_{i i k}^{\Re}+\hat{\boldsymbol{g}}_{i i k}^{\Im} \tan \theta\right]$. Now, constraints (C2a) and $(\mathrm{C} 2 \mathrm{~b})$ have been transformed into standard LMIs.

Now we turn to handle constraint (C2c). Introduce an auxiliary vector $\boldsymbol{t}_{i k}=\left[\varphi_{i k}, u_{i k}\right]^{T}$ to accommodate the variables $\varphi_{i k}$ and $u_{i k}$. Defining $\boldsymbol{U}_{i k}=\boldsymbol{t}_{i k} \boldsymbol{t}_{i k}^{H},(\mathrm{C} 2 \mathrm{c})$ is relaxed as 


$$
\left\{\begin{array}{l}
\operatorname{Pr}\left\{\left(\tilde{\boldsymbol{g}}_{i i k}^{\Re}\right)^{T} \boldsymbol{w}_{i}^{\Im}+\left(\tilde{\boldsymbol{g}}_{i k k}^{\Im}\right)^{T} \boldsymbol{w}_{i}^{\Re} \leq\left(\left(\tilde{\boldsymbol{g}}_{i i k}^{\Re}\right)^{T} \boldsymbol{w}_{i}^{\Re}-\left(\tilde{\boldsymbol{g}}_{i i k}^{\Im}\right)^{T} \boldsymbol{w}_{i}^{\Im}-\sqrt{\left(\sigma_{n}^{2}+\sum_{j \neq i}^{N_{B S}}\left\|\tilde{\boldsymbol{g}}_{j i k}^{T} \boldsymbol{w}_{j}\right\|^{2}\right) \bar{\Gamma}_{i k}}\right) \tan \theta \mid \boldsymbol{e}_{i k}\right\} \geq \eta_{i k}, \\
\operatorname{Pr}\left\{-\left(\tilde{\boldsymbol{h}}_{i i k}^{\Re}\right)^{T} \boldsymbol{w}_{i}^{\Im}-\left(\tilde{\boldsymbol{h}}_{i i k}^{\Im}\right)^{T} \boldsymbol{w}_{i}^{\Re} \leq\left(\left(\tilde{\boldsymbol{g}}_{i i k}^{\Re}\right)^{T} \boldsymbol{w}_{i}^{\Re}-\left(\tilde{\boldsymbol{g}}_{i i k}^{\Im}\right)^{T} \boldsymbol{w}_{i}^{\Im}-\sqrt{\left(\sigma_{n}^{2}+\sum_{j \neq i}^{N_{B S} S}\left\|\tilde{\boldsymbol{g}}_{j i k}^{T} \boldsymbol{w}_{j}\right\|^{2}\right) \bar{\Gamma}_{i k}}\right) \tan \theta \mid \boldsymbol{e}_{i k}\right\} \geq \eta_{i k} .
\end{array}\right.
$$

$$
\left\{\begin{array}{l}
(C 2 a): \operatorname{Pr}\left\{\left[\hat{\boldsymbol{g}}_{i i k}^{\Im}+\boldsymbol{e}_{i k}^{\Im}-\hat{\boldsymbol{g}}_{i i k}^{\Re} \tan \theta-\boldsymbol{e}_{i k}^{\Re} \tan \theta ; \hat{\boldsymbol{g}}_{i i k}^{\Re}+\boldsymbol{e}_{i k}^{\Re}+\hat{\boldsymbol{g}}_{i i k}^{\Im} \tan \theta+\boldsymbol{e}_{i k}^{\Im} \tan \theta\right]^{T}\left[\boldsymbol{w}_{i}^{\Re} ; \boldsymbol{w}_{i}^{\Im}\right] \leq-\sqrt{\bar{\Gamma}_{i k}}\left(\sigma_{n}+\varphi_{i k}\right) \tan \theta\right\} \geq \eta_{i k}, \\
(C 2 b): \operatorname{Pr}\left\{\left[-\hat{\boldsymbol{g}}_{i i k}^{\Im}-\boldsymbol{e}_{i k}^{\Im}-\hat{\boldsymbol{g}}_{i k}^{\Re} \tan \theta-\boldsymbol{e}_{i k}^{\Re} \tan \theta ;-\hat{\boldsymbol{g}}_{i i k}^{\Re}-\boldsymbol{e}_{i k}^{\Re}+\hat{\boldsymbol{g}}_{i i k}^{\Im} \tan \theta+\boldsymbol{e}_{i k}^{\Im} \tan \theta\right]^{T}\left[\boldsymbol{w}_{i}^{\Re} ; \boldsymbol{w}_{i}^{\Im}\right] \leq-\sqrt{\bar{\Gamma}_{i k}}\left(\sigma_{n}+\varphi_{i k}\right) \tan \theta\right\} \geq \eta_{i k}, \\
(C 2 c): \varphi_{i k}^{2} \geq u_{i k},(C 2 d): u_{i k} \geq \sum_{j \neq i}^{N_{B S}}\left\|\tilde{\boldsymbol{g}}_{j i k}^{T} \boldsymbol{w}_{j}\right\|^{2} .
\end{array}\right.
$$

$$
(C 2 a):\left[\begin{array}{cc}
\frac{\left(-\overline{\boldsymbol{f}}_{i k}^{T}\left[\boldsymbol{w}_{i}^{\Re} ; \boldsymbol{w}_{i}^{\Im}\right]-\left(\sigma_{n}+\varphi_{i k}\right) \sqrt{\bar{\Gamma}_{i k}} \tan \theta\right) \boldsymbol{I}}{\Phi^{-1}\left(\eta_{i k}\right)} & \boldsymbol{\Lambda}_{i k}^{\frac{1}{2}}\left[\boldsymbol{w}_{i}^{\Re} ; \boldsymbol{w}_{i}^{\Im}\right] \\
\left(\boldsymbol{\Lambda}_{i k}^{\frac{1}{2}}\left[\boldsymbol{w}_{i}^{\Re} ; \boldsymbol{w}_{i}^{\Im}\right]\right)^{T} & \frac{-\overline{\boldsymbol{f}}_{i k}^{T}\left[\boldsymbol{w}_{i}^{\Re} ; \boldsymbol{w}_{i}^{\Im}\right]-\left(\sigma_{n}+\varphi_{i k}\right) \sqrt{\overline{\Gamma_{i k}} \tan \theta}}{\Phi^{-1}\left(\eta_{i k}\right)}
\end{array}\right] \succeq \mathbf{0} .
$$

$$
(C 2 b):\left[\begin{array}{cc}
\frac{\left(-\bar{d}_{i k}^{T}\left[\boldsymbol{w}_{i}^{\Re} ; \boldsymbol{w}_{i}^{\Im}\right]-\left(\sigma_{n}+\varphi_{i k}\right) \sqrt{\Gamma_{i k}} \tan \theta\right) I}{\Phi^{-1}\left(\eta_{i k}\right)} & \boldsymbol{\Lambda}_{i k}^{\frac{1}{2}}\left[\boldsymbol{w}_{i}^{\Re} ; \boldsymbol{w}_{i}^{\Im}\right] \\
\left(\boldsymbol{\Lambda}_{i k}^{\frac{1}{2}}\left[\boldsymbol{w}_{i}^{\Re} ; \boldsymbol{w}_{i}^{\Im}\right]\right)^{T} & \frac{-\overline{\boldsymbol{d}}_{i k}^{T}\left[\boldsymbol{w}_{i}^{\Re} ; \boldsymbol{w}_{i}^{\Im}\right]-\left(\sigma_{n}+\varphi_{i k}\right) \sqrt{\overline{\Gamma_{i k}} \tan \theta}}{\Phi^{-1}\left(\eta_{i k}\right)}
\end{array}\right] \succeq \mathbf{0},
$$

$$
(C 2 c): \operatorname{Tr}\left(\boldsymbol{\Xi} \boldsymbol{U}_{i k}\right)+\boldsymbol{l} \boldsymbol{t}_{i k} \geq 0 \text {; and }\left[\begin{array}{cc}
\boldsymbol{U}_{i k}, & \boldsymbol{t}_{i k} \\
\left(\boldsymbol{t}_{i k}\right)^{T}, & 1
\end{array}\right] \succeq \mathbf{0},
$$

where $\boldsymbol{\Xi}=\left[\begin{array}{ll}1, & 0 \\ 0 & 0\end{array}\right]$ and vector $\boldsymbol{l}=[0,-1]$. Evidently, (C2c) is a LMI constraint, and the last difficulty lies in (C2d). Expanding the right hand of $(\mathrm{C} 2 \mathrm{~d})$ yields

$$
\begin{aligned}
(C 2 d): & \sum_{j \neq i}^{N_{B S}}\left(\boldsymbol{e}_{i k}^{T} \boldsymbol{W}_{j} \boldsymbol{e}_{i k}^{*}\right)+\sum_{j \neq i}^{N_{B S}}\left(\boldsymbol{e}_{i k}^{T} \boldsymbol{W}_{j} \hat{\boldsymbol{g}}_{j i k}^{*}\right)+ \\
& \sum_{j \neq i}^{N_{B S}}\left(\hat{\boldsymbol{g}}_{j i k}^{T} \boldsymbol{W}_{j} \boldsymbol{e}_{i k}^{*}\right)+\sum_{j \neq i}^{N_{B S}}\left(\hat{\boldsymbol{g}}_{j i k}^{T} \boldsymbol{W}_{j} \hat{\boldsymbol{g}}_{j i k}^{*}\right)-u_{i k} \leq 0,
\end{aligned}
$$

To handle (C2d) with the presence of unknown CSI error $e_{i k}$. We first introduce S-Procedure in Lemma 1.

Lemma 1 (S-Procedure [18]): Define a function $y_{n}(\boldsymbol{x}), n \in$ $\{1,2\}$, as $y_{n}(\boldsymbol{x})=\boldsymbol{x}^{H} \boldsymbol{A}_{n} \boldsymbol{x}+2 \Re\left\{\boldsymbol{b}_{n}^{H} \boldsymbol{x}\right\}+c_{n}$, where $\boldsymbol{A}_{n} \in$ $\mathbb{H}^{N \times N}, \boldsymbol{b}_{n} \in \mathbb{C}^{N \times 1}$ and $c_{n} \in \mathbb{R}$. The implication $y_{1}(\boldsymbol{x}) \leq$ $0 \Rightarrow y_{2}(\boldsymbol{x}) \leq 0$ holds if and only if the following conditions hold

$$
\rho\left[\begin{array}{ll}
\boldsymbol{A}_{1}, & \boldsymbol{b}_{1} \\
\boldsymbol{b}_{1}^{H}, & c_{1}
\end{array}\right]-\left[\begin{array}{ll}
\boldsymbol{A}_{2}, & \boldsymbol{b}_{2} \\
\boldsymbol{b}_{2}^{H}, & c_{2}
\end{array}\right] \succeq \mathbf{0}, \text { and } \rho \geq 0 .
$$

Nevertheless, to incorporate (C2d) with S-Procedure, we first need to find the bound of $\boldsymbol{e}_{i k}^{T} \boldsymbol{I}_{M} \boldsymbol{e}_{i k}^{*}$ as a premise to implement S-Procedure. Since we know the distribution of the CSI error, we further introduce Lemma 2 to calculate the bound of the term $\boldsymbol{e}_{i k}^{T} \boldsymbol{I}_{M} \boldsymbol{e}_{i k}^{*}$, i.e. $\boldsymbol{e}_{i k}^{T} \boldsymbol{I}_{M} \boldsymbol{e}_{i k}^{*} \leq \nu^{2}$.

Lemma 2 (CSI Error Ellipsoidal Bound): Since the CSI error follows Gaussian distribution such that $\left[\boldsymbol{e}_{i k}\right]_{m} \sim \mathbb{C N}\left\{0, \sigma_{i k}^{2}\right\}$, $\forall m \in M$, the term $\boldsymbol{e}_{i k}^{H} \boldsymbol{I} \boldsymbol{e}_{i k} \leq \nu^{2}$ can be equivalently expressed as $\left(\frac{\left[e_{i k}\right]_{1}}{\sigma_{i k}}\right)^{2}+\ldots+\left(\frac{\left[e_{i k}\right]_{M}}{\sigma_{i k}}\right)^{2} \leq\left(\frac{\nu}{\sigma_{i k}}\right)^{2}$. The left hand follows chi-square distribution with freedom of size $M$. Hence, the term $\boldsymbol{e}_{i k}^{H} \boldsymbol{I} \boldsymbol{e}_{i k} \leq \nu^{2}$ can be approximately considered as the probability density function (pdf) of a chi-square variable such that $\operatorname{Pr}\left\{\left(\frac{\left[\boldsymbol{e}_{i k}\right]_{1}}{\sigma_{i k}}\right)^{2}+\ldots+\left(\frac{\left[\boldsymbol{e}_{i k}\right]_{M}}{\sigma_{i k}}\right)^{2} \leq \frac{\nu^{2}}{\sigma_{2 k}^{2}}\right\}=\delta . \delta$ denotes the probability of the inequality being satisfied, whose value can be set to close to 1 . Denoting $\Psi_{M}$ as the cdf of a chi-square distributed variable with degrees of freedom $M$, we have $\Psi_{M}\left\{\frac{\nu^{2}}{\sigma_{i k}^{2}}\right\}=\delta$. Denoting $\Psi_{M}^{-1}(\cdot)$ as the inverse function of $\Psi_{M}(\cdot)$, finally we get $\nu^{2}=\Psi_{M}^{-1}(\delta) \sigma_{i k}^{2}$.

Based on Lemma 2, we are able to obtain the value of $\nu^{2}$. Recalling S-procedure, we are able to utilize the LMI in (16) as a premise to guarantee the implication $\left(\boldsymbol{e}_{i k}^{T} \boldsymbol{I}_{M} \boldsymbol{e}_{i k}^{*}\right)-\nu^{2} \leq$ $0 \Rightarrow$ (14) holds. In summary, after a series of transformation, the original constraint $(\mathrm{C} 2)$ has been equivalently replaced by another four constraints (C2a)-(C2d), as shown by Eqs. (11), (12), (13) and (16). Defining precoding matrix $\boldsymbol{W}_{i}=\boldsymbol{w}_{i} \boldsymbol{w}_{i}^{H}$, the optimization problem can be finally given as

$$
\begin{aligned}
& P 2 \text { (Robust - CI - CBF) : } \underset{\boldsymbol{w}_{i}, \forall i \in \mathbb{N}_{B S}}{\operatorname{argmin}} \sum_{i=1}^{N_{B S}} \operatorname{Tr}\left(\boldsymbol{W}_{i}\right), \\
& \text { s.t }(C 1): \operatorname{Tr}\left(\boldsymbol{W}_{i}\right) \leq P_{\text {max }}, \forall i \in \mathbb{N}_{B S},(C 2 a):(11), \\
& \quad(C 2 b):(12),(C 2 c):(13),(C 2 d):(16), \forall \mathrm{U}_{i k}, \\
& (C 3):\left[\begin{array}{cc}
\boldsymbol{W}_{i} & \boldsymbol{w}_{i} \\
\boldsymbol{w}_{i}^{T} & 1
\end{array}\right] \succeq 0, \forall i \in \mathbb{N}_{B S}, \\
& (C 4): \operatorname{Rank}\left(\boldsymbol{W}_{i}\right)=1, \forall i \in \mathbb{N}_{B S} .
\end{aligned}
$$

where (C3) is the semi-definite relaxation version of the original equality $\boldsymbol{W}_{i}=\boldsymbol{w}_{i} \boldsymbol{w}_{i}^{H}, \forall i \in \mathbb{N}_{B S}$. For the rank-1 constraint in (C4), rank relaxation can be applied [19]. With the condition that the channel parameters are statistically independent assumed in this paper, the solution is of rank-one. Related proof has been extensively reported [19] [20], which is not provided in this paper due to page limit. Now, the problem is a standard 


$$
(C 2 d):\left[\begin{array}{cc}
\phi_{i k} \boldsymbol{I}_{M}-\sum_{j \neq i}^{N_{B S}} \boldsymbol{W}_{j}, & -\sum_{j \neq i}^{N_{B S}} \hat{\boldsymbol{g}}_{j i k}^{T} \boldsymbol{W}_{j} \\
-\left(\sum_{j \neq i}^{N_{B S} S} \hat{\boldsymbol{g}}_{j i k}^{T} \boldsymbol{W}_{j}\right)^{H}, & -\phi_{i k} \nu^{2}-\sum_{j \neq i}^{N_{B S}}\left(\hat{\boldsymbol{g}}_{j i k}^{T} \boldsymbol{W}_{j} \hat{\boldsymbol{g}}_{j i k}^{*}\right)+u_{i k}
\end{array}\right] \succeq \mathbf{0}, \text { and } \phi_{i k} \geq 0 .
$$

semi-definite programming (SDP) optimization, which can be readily solved by CVX.

\section{OVERHEAD AND COMPLEXITy ANALYSIS}

In this section, we analyze the incurred coordination overhead and the complexity of the proposed optimization.

\section{A. Coordination Signalling Overhead Analysis}

The proposed CI-CBF-Robust scheme utilizes multi-user interference while cancelling the negative effect of inter-cell interference. Hence, each BS only needs to share CSI with others, alleviating the requirement of sharing data. Assume that there are $K$ users in each BS. Given $N_{B S}$ coordinated BSs, the overall overhead for coordination can be given as $\mathcal{O}\left(N_{B S}^{2}\left(N_{B S}-1\right) K \chi_{C}\right)$. The variable $\chi_{C}$ represents the bits for sharing one user's channel vector, which is related to the size of channel vectors and the method adopted for describing CSI. In practice, BSs can use code-word for describing CSI, and the index of the selected code-word represents a predefined CSI, typically 2-10 bits long [21]. As can be seen, the proposed scheme incurs the same level of overhead with that of conventional $\mathrm{CBF}$ schemes, whereas maintaining the advantage of utilizing multiuser interference. On the contrary, the CoMP systems need to share both the CSI and the instantaneous intended data. Hence, the overall overhead of sharing CSI and data is given $\mathcal{O}\left(N_{B S}\left(N_{B S}-1\right) K\left(N_{B S} \chi_{C}+\chi_{S}\right)\right)$, where $\chi_{S}$ denotes the bits for exchanging instantaneous data, typical 280 bits in each type-2 LTE sub-frame. Evidently, the CoMP incurs much more overhead over the CI-based CBF systems, especially with a high number of coordinated BSs.

\section{B. Complexity Analysis}

Now we present the complexity of the proposed CI-CBFRobust scheme. Since the transformed optimization P3 is a standard SDP problem, CVX can be applied to solve it with interior-point method with analytical complexity $\ln \left(\frac{1}{\epsilon}\right) \sqrt{c_{b}}\left(c_{f}+c_{g}\right)$ [22]. In particular, the term $\ln \left(\frac{1}{\epsilon}\right)$ denotes the accuracy setup for algorithm convergence. $\sqrt{c_{b}}$ denotes the barrier parameter counting the geometric complexity of the constraints, while $c_{f}$ and $c_{g}$ denote the complexities on the generation and factorization of the matrices in the optimization problem. Due to page limit, we refer readers to [22] for details.

For the proposed algorithm, it has $N_{B S}$ LMIs (trace) in $(C 1)$ of size $1, N_{B S} K$ LMIs of size $2 M+1$ in $(C 2 a), N_{B S} K$ LMIs of size $2 M+1$ in $(C 2 b), N_{B S} K$ LMIs (trace) of size 1 and $N_{B S} K$ LMI constraint of size 3 in $(C 2 c), N_{B S} K$ LMI constraint of size $\left(N_{B S}-1\right) M^{2}+1$ and $N_{B S} K$ linear constraints in $(C 2 d)$, and finally $N_{B S} K$ LMIs of size $M+1$ in $(C 3)$. Hence, the overall complexity can be analytically evaluated as $\ln \left(\frac{1}{\epsilon}\right) \beta_{3}\left(n\left(N_{B S}+10 N_{B S} K+N_{B S} K\left(\left(N_{B S}-\right.\right.\right.\right.$ 1) $\left.\left.M^{2}+1\right)^{3}+2 N_{B S} K(2 M+1)^{3}+N_{B S} K(M+1)^{3}\right)+n^{2}\left(N_{B S}+\right.$

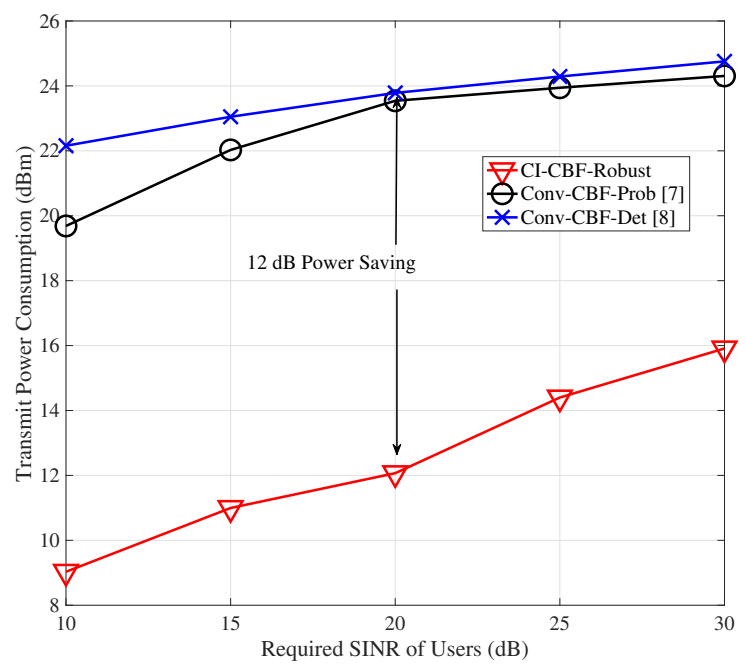

Fig. 2. The total power consumption of different algorithms vs. users' SINR requirements $\bar{\Gamma}_{i k}$, where $M=4$ antennas are equipped at each BS. $K=3$ users are randomly placed in each cell. CSI error $\sigma_{i k}=10^{-2}$ and power budget $P_{\max }=40 \mathrm{dBm}$.

$10 N_{B S} K+N_{B S} K\left(\left(N_{B S}-1\right) M^{2}+1\right)^{2}+2 N_{B S} K(2 M+$ $\left.\left.1)^{2}+N_{B S} K(M+1)^{2}\right)+n^{3}\right)$. Evidently, the proposed scheme has a polynomial complexity, confirming its practicality in optimization design.

\section{Simulation Results}

In this section, the performance of the proposed scheme is demonstrated. The classical CBF schemes are selected as benchmarks, where both multi-user and inter-cell interference are strictly mitigated. We selected the mostly related works in [7] and [8] as comparisons. The former (denoted as Conv-CBFProb) minimizes transmission power under probabilistic SINR constraints, while the latter (denoted as Conv-CBF-Det) guarantees SINR constraints by deterministic robust optimization. The central frequency is set to $2 \mathrm{GHz}$ with $1 \mathrm{MHz}$ signal bandwidth. Following the closely relevant works in [7] [8], we consider a 3-hexagon-cell coordination scenario. A BS equipped with $M=4$ antennas is deployed in the center of each cell. $K=3$ users are randomly placed in each cell. The transmission power budget at each BS is set to $P_{\max }=40 \mathrm{dBm}$. QPSK is selected for signal constellation. We assume a practical CSI quality such that $\sigma_{i k}=10^{-2}$. The probability of satisfying the preset SINR requirement $\left(\bar{\Gamma}_{i k}=20 \mathrm{~dB}\right)$ is $\eta_{i k}=80 \%$. The power spectral density of AWGN is $-174 \mathrm{dBm} / \mathrm{Hz}$. The large-scale and Rayleigh small-scale fading models in [7] are selected for modelling the channels. 


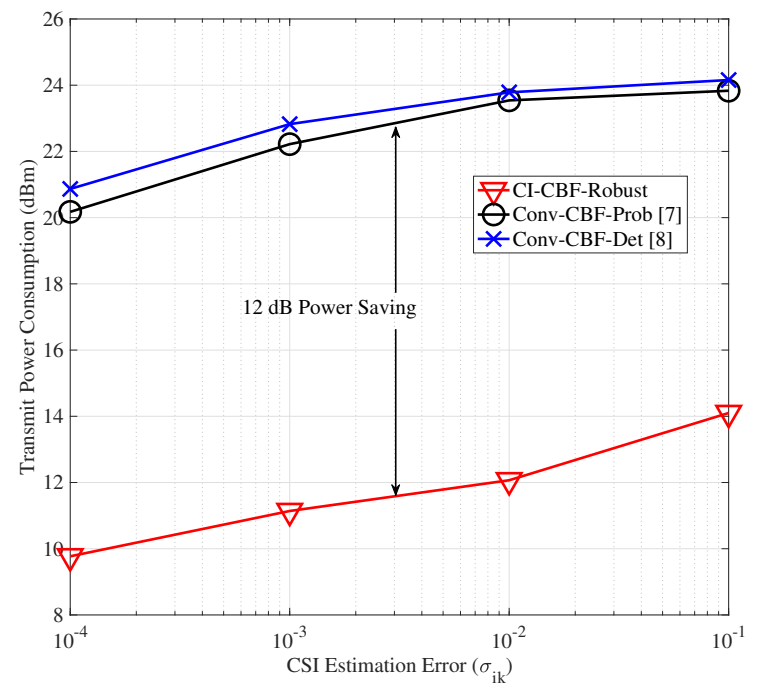

Fig. 3. The total power consumption of different algorithms vs. the accuracy of channel estimation, where SINR requirement is $\bar{\Gamma}_{i k}=20 \mathrm{~dB} . M=4$ antennas are equipped at each BS. $K=3$ users are randomly placed in each cell and power budget $P_{\max }=40 \mathrm{dBm}$.

Fig. 2 shows the transmission power consumption of different algorithms under users' SINR requirements $\bar{\Gamma}_{i k}$. It can be observed that the proposed CI-CBF-Robust scheme saves 8$12 \mathrm{~dB}$ power consumption over the benchmarks. It is because the proposed algorithm effectively utilizes multiuser interference as a desired element rather than cancelling it, which is fundamentally different from the conventional CBF scheme that always treats intra-cell multiuser interference as destructive noise. Hence, to achieve a pre-set SINR target, the conventional CBF schemes have to dissipate more transmission power.

Fig. 3 shows the effect of the accuracy of CSI estimation on the transmission power. It is seen that a higher variance of CSI error leads to more power consumption. It is because a higher CSI variance increases the norm of covariance matrix $\boldsymbol{\Lambda}_{i k}$. Hence, based on Eqs. (11) and (12), the amplitude of the precoder should be correspondingly increased to guarantee the semi-definite property of the matrices. As a result, higher transmission power is also dissipated. Still, it can be observed with different levels of CSI estimation accuracy, the proposed scheme provides up to $12 \mathrm{~dB}$ power saving compared to the benchmark, showing a high robustness against the CSI error.

\section{CONCLUSIONS}

In this paper, we have presented a novel CBF coordination scheme to judiciously utilize multi-user interference as constructive components while simultaneously mitigating intercell interference, based on the shared CSI among the coordinated BSs. Taking imperfect CSI into consideration, we have minimized transmission power under users' probabilistic SINR constraints, which guarantees the users' SINR requirements in a statistical manner. The simulation results have verified that, the proposed CI-CBF-Robust algorithm saves 8-12 dB transmission power over the benchmarks, and shows the highest level of robustness against CSI error. In addition, the overhead and complexity of the proposed scheme are analytically analyzed, confirming its high practicality in multi-cell coordination.

\section{REFERENCES}

[1] H. Zeng et al., "A green coordinated multi-cell NOMA system with fuzzy logic multi-criterion user mode selection and resource allocation," IEEE Sel. Topics Sig. Process., vol. 13, no. 3, pp. 480-495, Jun. 2019.

[2] D. Lee et al. "Coordinated multipoint transmission and reception in LTE-advanced: deployment scenarios and operational challenges," IEEE Commu. Mag., vol. 50, no. 2, pp. 148-155, Feb. 2012.

[3] F. Wang, "Joint user association, subchannel allocation, and power allocation in multi-cell multi-association OFDMA heterogeneous networks," IEEE Trans. Commun., vol. 65, no. 6, pp. 2672-2684, Jun. 2017.

[4] S. Ararat and M. R. Nakhai, "Robust distributed beamforming with interference coordination in downlink cellular networks," IEEE Trans. Commun., vol. 62 no. 7, pp. 2411-2421, Jul. 2014.

[5] K. Huq et al., "Green HetNet: energy efficiency analysis and optimization," IEEE Trans. Veh. Technol., vol. 64, no. 10, pp. 670-683, Oct. 2015.

[6] P. Komulainen, A. Tolli, and M. Juntti, "Effective CSI signaling and decentralized beam coordination in TDD multi-cell MIMO systems," IEEE Trans. Signal Process., vol. 61, no. 9, pp. 2204-2218, May 2013.

[7] S. Nasseri, M. R. Nakhai, and T. A. Le, "Chance constrained robust downlink beamforming in multicell networks," IEEE Trans. Mobile. Comput., vol. 15, no. 11, pp. 2682-2691, Nov. 2016.

[8] C. Shen et al., "Distributed robust multi-cell coordinated beamforming with imperfect CSI: an ADMM approach,' IEEE Trans. Sig. Proc., vol. 60, no. 6, pp. 2988-3003, Jun. 2012.

[9] D. H. N. Nguyen and T. L. Ngoc, "Sum-rate maximization in the multicell MIMO multiple-access channel with interference coordination," IEEE Trans. Wireless Commun., vol. 13, no. 1, pp. 36-48, Jan. 2014.

[10] H. Wu, X. Gao, X. Wang, and X. You, "Sum-rate-optimal precoding for multi-cell large-scale MIMO uplink based on statistical CSI," IEEE Trans. Commun., vol. 63, no. 8, pp. 2924-2935, Aug. 2015.

[11] C. Masouros and E. Alsusa, "A novel transmitter-based selective precoding technique for DS/CDMA systems," IEEE Sig. Process. Lett., vol. 14, no. 9, pp. 637-640, Sep. 2007.

[12] D. Spano, M. Alodeh, S. Chatzinotas, and B. Ottersten, "Symbol level precoding for the non-linear multiuser MISO downlink channel," IEEE Trans. Signal Process., vol. 66, no. 5, pp. 1331-1345, Mar. 2018.

[13] Z. Wei and C. Masouros, "User-centric distributed antenna transmission: secure precoding and antenna selection with interference exploitation," IEEE Internet Things J., vol. 7, no. 3, pp. 2293-2308, Mar. 2020.

[14] K. L. Law, C. Masouros, and M. Pesavento, "Transmit precoding for interference exploitation in the underlay cognitive radio Z-channel," IEEE Trans. Signal Process., vol. 65, no. 14, pp. 3617-3630, Jul. 2017.

[15] Z. Wei et al., "Energy- and cost-efficient physical layer security in the era of IoT: the role of interference," IEEE Commun. Mag., DOI: 10.1109/MCOM.001.1900716.

[16] C. Masouros et al., "Known interference in the cellular downlink: a performance limiting factor or a source of green signal power?" IEEE Commun. Mag., vol. 51, no. 10, pp. 162-171, Oct. 2013.

[17] IEEE 802.16 Broadband Wireless Access Working Group. [2007, Nov] Channel estimation modeling for system simulations, [Online] Available: http://ieee802.org/16/tgm/contrib/C80216m-07_208r2.pdf

[18] S. Boyd and L. Vandenberghe, Convex Optimization. Cambridge, U.K.: Cambridge Univ. Press, 2004.

[19] Z. Luo, W. K. Ma, A. M. So, Y. Ye, and S. Zhang, "Semi-definite relaxation of quadratic optimization problems," IEEE Signal Process. Mag., vol. 27, issue. 3, pp. 20-34, May 2010.

[20] D. Nguyen, L. N. Tran, and M. L. Aho, "Precoding for full duplex multiuser MIMO systems: Spectral and energy efficiency maximization," IEEE Trans. Signal Process., vol. 61, no. 16, pp. 4038-4050,

[21] P. Huang and Y. Pi, "A novel MIMO channel state feedback scheme and overhead calculation," IEEE Trans. Commun., vol. 16, no. 10, pp. 4550-4562, Oct. 2018.

[22] K. Wang, W. Ma, and C. Chi, "Outage constrained robust transmit optimization for multiuser MISO downlinks: tractable approximations by conic optimization," IEEE Trans. Antenna Propagat., vol. 62, no. 21, pp. 5690-5715, Nov. 2014. 